\title{
IL-2/Lptn Gene-Modified Allogeneic Neuroblastoma Tumor Cell Vaccine
}

National Cancer Institute

\section{Source}

National Cancer Institute. IL-2/Lptn Gene-Modified Allogeneic Neuroblastoma Tumor

Cell Vaccine. NCI Thesaurus. Code C78487.

A cancer vaccine consisting of allogeneic neuroblastoma tumor cells have been genetically modified to secrete the human cytokine interleukin-2 (IL-2) and the human chemokine lymphotactin (Lptn) with potential immunostimulating and antineoplastic activities. Upon administration, IL-2 and Lptn are secreted by the IL-2/Lptn gene-modified allogeneic neuroblastoma tumor cell vaccine, potentially enhancing the cytotoxic T lymphocyte $(\mathrm{CT} L)$ response elicited by vaccine neuroblastoma tumor-associated antigens (TAAs) against host neuroblastoma tumor cells. Produced by activated progenitor T cells, Lptn belongs to the C chemokine subfamily and is a potent chemotactic factor for lymphocytes; IL-2 stimulates natural killer (NK) cells and may enhance a vaccine-elicited CTL immune response against tumor cells. 